\title{
Bone Motion Analysis from Dynamic MRI: Acquisition and Tracking
}

\author{
Benjamin Gilles ${ }^{1}$, Rosalind Perrin ${ }^{2}$, Nadia Magnenat-Thalmann ${ }^{1}$, and \\ Jean-Paul Vallée ${ }^{2}$ \\ 1 MIRALab, University of Geneva, CH-1211 Geneva, Switzerland, \\ \{gilles, thalmann\}@miralab.unige.ch \\ ${ }^{2}$ Geneva University Hospital, Radiology department, CH-1205 Geneva, Switzerland,
}

\begin{abstract}
For diagnosis, preoperative planning and postoperative guides, an accurate estimate of joints kinematics is required. We bring together MRI developments and new image processing methods in order to automatically extract active bone kinematics from multi-slice realtime dynamic MRI. We introduce a tracking algorithm based on 2D/3D registration and a procedure to validate the technique by using both dynamic and sequential MRI. We present how we optimize jointly the tracking method and the acquisition protocol to overcome the trade-off in acquisition time and tracking accuracy. As a case study, we apply this methodology on the human hip joint.
\end{abstract}

\section{Introduction}

Periacetabular osteotomy is an accepted surgical procedure to reorient the acetabulum in patients with hip symptoms of mechanical overload, impingement or femoral head instability. For both the diagnosis and the surgical planning, an accurate estimate of hip joint bone motion is required. Orthopedists can use animated 3D models, prior to joints surgeries, to evaluate their task and generally to reduce the overall time of the surgical operation. The long-term objective of our ongoing project is to model, analyze and visualize human joint motion in-vivo and non-invasively.

In order to deduce kinematical properties of the musculoskeletal system, techniques have been developed to measure internal motion of organs. The use of bone screws or implantable markers [1] provides a gold standard of bone motion measurement, although it is a very invasive approach. Optical motion capture consisting in recording markers trajectories attached to the skin leads to inaccuracy in the estimation of the position of internal organs because of fat/skin sliding artifacts [2]. Nowadays, medical imaging technology has reached a level where it is possible to capture internal motion with different modalities (CT, MRI, US). Several authors have reported kinematic studies of joints with sequential MRI acquisition techniques to evaluate the joint under passive motion, meaning the joint is stationary during acquisition. Brossmann et al. 3] reported the importance of acquiring joint motion actively, due to the existence of statistically significant variations between acquiring actively or passively. However, 
the problem of acquiring volumetric image data in real-time with MRI during active motion remains to be solved due to inherent trade-off in the MR imaging technique between Signal-to-Noise Ratio (SNR), spatial resolution and temporal resolution. Quick et al 4 published results on the use of the trueFISP (or b-FFE, FIESTA) imaging sequence for real-time imaging of active motion of the hand, ankle, knee and elbow (matrix 135 X 256, 6 frames/s) on a single slice. Bone motion tracking in 2D dynamic images, which are incomplete from a spatial point of view, is equivalent to a $2 \mathrm{D} / 3 \mathrm{D}$ rigid registration between dynamic images and the static MRI volume used to reconstruct 3D models. Various registration methods have been proposed in the literature [5]. 2D/3D multimodal rigid registration has been investigated for intra-operative navigation using mainly X-rays and CT data. Tomazevic et al. 6] presented a technique based on bone surface matching; Zöllei et al. [7], a method based on mutual information optimization.

This paper presents the selection of the best dynamic imaging protocol available to our group and the adaptation of the technique to the joint motion extraction problem. We introduce a new technique to track bone motion automatically from real-time dynamic MRI based on the combination of temporal information of dynamic MRI and spatial information of static MRI by $2 \mathrm{D} / 3 \mathrm{D}$ registration. Bone motion tracking in sequential MRI is used as a gold standard bone position measurement. Subsequently, we present how we optimized both the tracking method and the acquisition protocol to overcome the trade-off in acquisition time and tracking accuracy.

\section{Real-Time Dynamic Images Acquisition}

\subsection{In Vitro Study}

The acquisition was performed with a $1.5 \mathrm{~T}$ Intera MRI system (Philips Medical Systems, Best NL). In a first step, the b-FFE (balanced Fast Field Echo, Philips Medical Systems, Best NL) imaging sequence (aka. trueFISP) was quantitatively compared to four other sequences, including Turbo Spin Echo (TSE), RF-spoiled FFE (T1-FFE) and a Field Echo, Echo Planar Imaging (FE-EPI) sequence. In order to quantify sequence performance, a phantom consisting of tubes of Gd-DTPA (Schering AG, Germany) at varying concentrations was used. Using this phantom, measurements of SNR could be made for a range of physiological T2/T1 values. The b-FFE sequence was found to outperform all other ultra-fast MR sequences available on the scanner in terms of SNR divided by the acquisition time, SNRt. The SNR and CNR (between muscle and fat) was optimal at a flip angle of 90deg for b-FFE sequence. Partial Fourier acquisition in the read-out direction was possible without significant reduction in image quality. This enabled the scan time to be reduced by $30 \%$.

\subsection{In Vivo Study}

The imaging protocol was developed and optimized with reference to the limitations of the tracking algorithm. First, the trade off in image quality with 
FOV and matrix was investigated qualitatively on healthy volunteers in order to achieve the optimum resolution, contrast and frame acquisition time. As scan duration was proportional to the phase encode matrix, the phase encode matrix was maintained $<100$ at the shortest repetition time possible (TR $3.5 \mathrm{~ms}$ ). It was found that reducing the FOV and hence the phase encode matrix, maintaining an in-plane resolution of $2 \mathrm{~mm}$, was not an effective way to reduce frame acquisition time, due to the need to use fold-over suppression to avoid aliasing in the phase encode direction. A parallel imaging technique, SENSE (Philips Medical Systems, Best NL), was found to reduce the scan time by a factor of 2 without significant reduction in image quality. A reference scan is acquired prior to the SENSE MR sequence to measure the sensitivity profile of the phased-array coil. The same reference scan is used for all the images of the dynamic series.

A positioning device was developed that facilitated reproducible abductive motion in both sequential and dynamic modes. A study was run with six healthy volunteers to optimize and evaluate the robustness of the registration-MRI protocol combination without the introduction of motion artifacts. Ethics approval was obtained from the local ethics committee for the study protocol. In a first session a complete static image data set of the pelvis and femur was acquired with a 2D multi-slice spin echo acquisition (TR/TE 578/18ms). In the second scan session the joint was stepped successively in abduction, and at a range of positions two scans were run. A 3D sequential acquisition at high spatial resolution (fast gradient echo sequence with radial reconstruction: FFE, TR/TE $6.4 / 3.1 \mathrm{~ms}$, Flip angle $15 \mathrm{deg}$, FOV/matrix $500 \mathrm{~mm} / 410 \times 512$ ) was run to localize the hip position (gold standard) and secondly the optimized 2D dynamic protocol was run (seven imaging planes, gradient echo sequence with balanced gradients: bFFE, TR/TE 3.5/1.1ms, Flip angle $80 \mathrm{deg}$, pixel size 2 x $2 \mathrm{~mm}$, slice thickness $10 \mathrm{~mm}$, partial Fourier reduction factor of 0.65 in read direction). The slice positions of the dynamic slices were required to be adjusted to intersect appropriate bony landmarks on each volunteer. These planes were set initially and maintained throughout the sequential motion protocol.

\section{Bone Motion Tracking}

\subsection{Mathematical Definitions}

Prior to tracking, the femur and the pelvis are automatically segmented and reconstructed from the static image data (three volumes rigidly registered in the static coordinate system $W_{\mathrm{s}}$ ) using a deformable model-based method presented in [8] (see Fig. 1). We use a 3D simulation method based on bone-to-bone collision detection (see 9] for more details) to determine a fixed hip joint center of rotation $C$. Standard orthogonal coordinate systems of the femur $\left(S_{\mathrm{f}}\right)$ and the pelvis $\left(S_{\mathrm{p}}\right)$ are centered on $C$ and oriented using anatomical landmarks [10] (see Fig.1). Let $M_{\mathrm{f}}=M_{\left(\mathrm{S}_{\mathrm{f}} \rightarrow \mathrm{W}_{\mathrm{s}}\right)}\left(\operatorname{resp} . M_{\mathrm{p}}=M_{\left(\mathrm{S}_{\mathrm{p}} \rightarrow \mathrm{W}_{\mathrm{s}}\right)}\right)$ be the corresponding homogeneous transformation matrices.

The bone tracking problem is equivalent to rigidly registering at each instant $t$ the $3 \mathrm{D}$ static volume where bony regions have been segmented and the $2 \mathrm{D}$ 

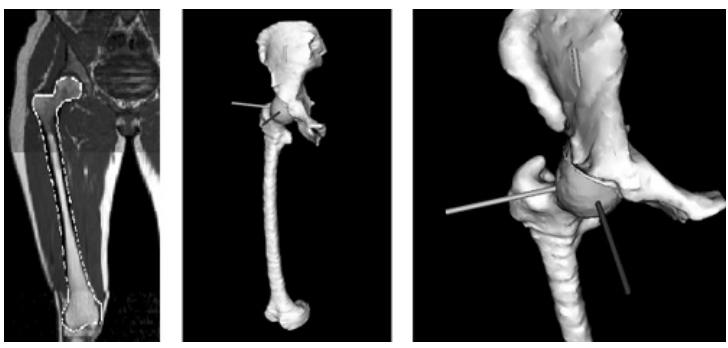

Fig. 1. Results of the automatic femur segmentation on a sample slice, reconstructed $3 \mathrm{D}$ models and standard coordinate system of the pelvis.

dynamic planes. A registration problem can often be stated as a functional energy minimization. The energy, calculated with a similarity metric [11, measures how good the matching is. Let $\theta_{\mathrm{t}}^{\mathrm{f}}\left(\right.$ resp. $\theta_{\mathrm{t}}^{\mathrm{p}}$ ) be the six registration parameters for translations and rotations of the femur (resp. the pelvis). Dynamic plane relative positions are defined from the acquisition parameters as a set of $N$ (number of planes) coordinate systems $P_{\mathrm{i}}(i \in[0 . ., N])$ in the dynamic acquisition system $W_{\mathrm{d}}$ corresponding to the homogeneous matrices $O_{\mathrm{i}}=M_{\left(\mathrm{P}_{\mathrm{i}} \rightarrow \mathrm{W}_{\mathrm{d}}\right)}$. We define a transformation $\phi^{\mathrm{f}}: \Re^{2} X \aleph \rightarrow \Re^{3}$ (resp. $\phi^{\mathrm{p}}: \Re^{2} X \aleph \rightarrow \Re^{3}$ ) that maps a point of the plane $\mathrm{z}=0$ in $P_{\mathrm{i}}$ to a point in $W s$ for the femur (resp. the pelvis) such as $(\operatorname{method} \mathrm{A})$ :

$$
\phi_{\theta_{\mathrm{t}}^{\mathrm{f}}}^{\mathrm{f}}(x, y, i)=M_{\mathrm{f}} \cdot Q_{\mathrm{t}}^{\mathrm{f}} \cdot O_{\mathrm{i}} \cdot[x, y, 0,1]^{\mathrm{T}} \text { and } \phi_{\theta_{\mathrm{t}}^{\mathrm{p}}}^{\mathrm{p}}(x, y, i)=M_{\mathrm{p}} \cdot Q_{\mathrm{t}}^{\mathrm{p}} \cdot O_{\mathrm{i}} \cdot[x, y, 0,1]^{\mathrm{T}}
$$

$Q_{\mathrm{t}}^{\mathrm{f}}=M_{\left(\mathrm{W}_{\mathrm{d}} \rightarrow \mathrm{S}_{\mathrm{f}}\right)}\left(\operatorname{resp} \cdot Q_{\mathrm{t}}^{\mathrm{p}}=M_{\left(\mathrm{W}_{\mathrm{d}} \rightarrow \mathrm{S}_{\mathrm{p}}\right)}\right)$ is defined by $\theta_{\mathrm{t}}^{\mathrm{f}}\left(\operatorname{resp} . \theta_{\mathrm{t}}^{\mathrm{p}}\right)$ using unit quaternions formulation for rotations [12]. It represents the position of the femur (pelvis) in $W_{\mathrm{d}}$. $\phi$ can be expressed in different ways. For instance, we can use the relative position between the femur and the pelvis such as $Q_{\mathrm{t}}^{\mathrm{rel}}=Q_{\mathrm{t}}^{\mathrm{p}}\left(Q_{\mathrm{t}}^{\mathrm{f}}\right)^{-1}$ (method B). In this case, $\theta_{\mathrm{t}}^{\text {rel }}$ (defining $Q_{\mathrm{t}}^{\mathrm{rel}}$ ) are the registration parameters. The conversion of $Q_{\mathrm{t}}^{\mathrm{rel}}$ into standard hip joint angles gives normalized flexion, adduction and internal rotation angles which are medically relevant angles. Another way to represent $\phi$ is to use the variation of the relative transformations between the femur and the pelvis from one frame to the next one: $Q_{\mathrm{t}}^{\mathrm{diff}}=\left(Q_{\mathrm{t}-1}^{\mathrm{rel}}\right)^{-1} Q_{\mathrm{t}}^{\mathrm{rel}}$ (method C).

The similarity metric aims at measuring the degree of alignment between the reference dataset (static MRI volume) and the transformed dataset (dynamic MRI images). In case of MR images, no similarity metric has proven to be superior especially when using different acquisition protocols with different tissues/intensity transfer functions. Roche et al. showed the importance of choosing an appropriate metric 13. We have implemented three standard similarity metrics [1]: normalized cross-correlation (NCC), absolute differences (AD) and mutual information (MI). In addition, we use a metric we call "model matching" 
(MM) that measures, independent of the static volume, the alignment of the reconstructed model and the edges of the dynamic images. Also, NCC, AD and MM are applied to the gradient vector images and are denoted by GNCC, GAD and GMM. Grey-scale values in the static volume are trilinearly interpolation at floating positions defined by the transformed dynamic images. The similarity is performed in the bone neighborhood where the motion is purely rigid. In other words, soft tissues that deform significantly are ignored. Considering a bone model reconstructed from the static MRI volume, we define a mask (subset of the static volume) where locations are inside the model or at a distance, determined empirically, of $5 \mathrm{~mm}$ from its surface. The mask is automatically generated using the ICP (Iterative Closest Point) algorithm.

\subsection{Registration Procedure}

The hip bone tracking problem to be resolved is to find, at each instant $t$ and for each bone, the solution parameters $\theta_{\mathrm{t}}^{*}$ that minimize the similarity measurement between the static volume and the transformed dynamic images. We can use either method A, B or C to define the rigid transformation. Given an optimization method $\Psi$ and a solution search space $\Theta$, we have:

$$
\theta_{\mathrm{t}}^{*}=\operatorname{argmin}_{\left(\theta_{\mathrm{t}} \in \Theta \mid \Psi\right)} \Delta\left(\phi_{\theta_{\mathrm{t}}}\left(D_{\mathrm{r}, \mathrm{t}}\right), S_{\mathrm{r}}\right)
$$

A coarse initialization is done manually. We use the amoeba optimizer, which is an implementation of the Nelder-Mead method 14 derived from simplex algorithm, as it is parameterizable (the number of iterations and the scale used when a parameter is modified can be set) and relatively robust in presence of local solutions. The three transformation parameters for rotations are the angle of the unit quaternion $q$ defining $Q_{\mathrm{t}}$ and two orthogonal components used to modify the vectorial part of $q$.

Tracking bones in a real-time sequences, yields to the question of the initialization: how to provide an accurate initialization for a particular frame $t$, knowing bones position in the preceding ones? The pelvis remains nearly immobile during movement implying that the user initialization for the first frame is suitable for the others. As a first step, we use method A to track the pelvis as it is independent to the position of the femur. To initialize the femur, we make the assumption that the movement is uniform. We tested two different initializations that led to comparable results: the spherical quaternion interpolation (so-called Slerp [12) for $Q_{\mathrm{t}}^{\text {rel }}$ using frames $t-1$ and $t-2$ with an interpolation parameter equals to 2 , and the use of the variation of relative transformation such as $Q_{\mathrm{t}}^{\text {diff }}=Q_{\mathrm{t}-1}^{\mathrm{diff}}$. The tracking is done using method C as it is more convenient for the optimization. More precisely, if the motion of the femur with regards to the pelvis is planar, which is roughly correct, only one optimization parameter (quaternion angle $\Omega$ ) defining $Q_{\mathrm{t}}^{\text {diff }}$ is modified. Obviously, for frames 0 and 1 , where we cannot use method $\mathrm{C}$ formulation as it depends on $t-2$ frame, we use method B. 


\section{Results}

\subsection{Tracking in Sequential MRI}

3D sequential acquisition gives a gold standard of bone positioning as it provides high spatial resolution. Because of acquisition time constraints, the sequential acquisition protocol (fast) is different to the initial static acquisition protocol. Bones tracking is done in two steps. First, bones positions are initialized for the first frame $t=0$ assuming that there is no translation of the hip joint center (HJC) and using GMM metric which is computationally fast. At $t=0$, the subject is in a neutral position (near zero position) and we have a good confidence that the HJC (estimated with method [9]) is correct as zero position is the reference for this calculation. It is corroborated by visual inspection of the alignment between bones contours in sequential MRI and 3D models. Second, the sequential volume at $t=0$ is used as the reference (static) volume to track bones in the other frames, with AD metric. AD metric is accurate in this case because contrasts are the same. Translation parameters of the relative position between the pelvis and the femur represent the translation of the estimated HJC. Over 46 different positions (36 abductions, 5 flexions and 5 internal/external rotations) and 6 different subjects, the average translation is $0.53 \mathrm{~mm}$ (standard deviation $=0.4 \mathrm{~mm}$, maximum $=2.4 \mathrm{~mm}$ ). It shows that the error in estimating the HJC (cumulated with possible translation of the real HJC) is minor.

\subsection{Optimization and Validation of the Method}

To measure the goodness of the tracking in dynamic MRI and hence to validate it, we compared, for a fixed subject position, pelvis/femur relative positions tracked in dynamic MRI with the ones tracked in sequential acquisition. The difference provides, similarly to [13, errors in rotation and translation. By minimizing these errors, we optimized tracking parameters. We determined empirically the parameters of the amoeba optimization procedure: scale of $1 \mathrm{~mm}$ for translations, scales of $0.05 \mathrm{~mm}$ and $0.05 \mathrm{rad}$ for rotations (defined with quaternions) and 200 iterations. We compared the seven different similarity metrics that we have implemented, keeping the same initial conditions (seven imaging planes, same initialization and same optimization parameters). For the mutual information metric, we estimated probability densities by using the joint histogram with 1000 random samples and 32 intensity bins in the range of 0-255. We found that normalized cross-correlation based on gradient vector images performs the best tracking in terms of accuracy: mean error in translation $=1.8 \mathrm{~mm}$ ( standard deviation $=1 \mathrm{~mm})$, mean error in rotation $=1.3 \mathrm{deg}$ (standard deviation $=0.7 \mathrm{deg}$ ). Also it was found to be the most robust metric (the variation of the similarity around the solution was the sharpest).

In order to speed up the dynamic acquisition time, it is important to select the smallest number of planes and the smallest resolution that still preserve a acceptable accuracy (3deg of error in rotation). We measured the accuracy of the tracking (with the same tracking parameters) for all combinations of three planes 
from the initial configuration of seven imaging planes. For the tested abductive motion, optimal planes pass near the HJC and are approximately orthogonal as shown in Fig. 2. The mean error in translation is $2.4 \mathrm{~mm}$ (standard deviation = $1 \mathrm{~mm}$ ) and the mean error in rotation is $2.1 \mathrm{deg}$ (standard deviation $=1.1 \mathrm{deg}$ ). With this configuration, we simulated different resolutions by gaussian filtering and subsampling dynamic grey-scale images. A resolution of $4 \mathrm{x} 4 \mathrm{~mm}$ was found to be the limit: mean error in translation $=3.3 \mathrm{~mm}$ ( standard deviation $=1.7 \mathrm{~mm})$, mean error in rotation $=3.3 \mathrm{deg}($ standard deviation $=1.5 \mathrm{deg})$.

\subsection{Application on Real-Time Dynamic MRI}

We applied our method on real-time dynamic sequences (with motion artifacts) and obtained visually satisfactory results. The dynamic protocol was a fast gradient echo sequence with balanced gradients (bFFE, TR/TE 3.5/1.1ms, Flip angle $80 \mathrm{deg}$, pixel size $4.7 \times 2.6 \mathrm{~mm}$, partial Fourier reduction factor of 0.65 in read direction, SENSE acceleration factor of 2 , frame rate $=6.7$ frames $/ \mathrm{s}$ ). This protocol provides sufficient morphological data for bone tracking to be carried out. For the optimization we used the parameters: GNCC metric, 200 iterations, $1 \mathrm{~mm}$ for the translation scale, $0.05 \mathrm{~mm}$ and $0.05 \mathrm{rad}$ for rotation scales. In case of a free abductive motion, with no positioning device, it was difficult to constrain the femur to remain in the coronal plane. Hence we used four planes by adding another coronal plane parallel to the previous one (Fig. 2).
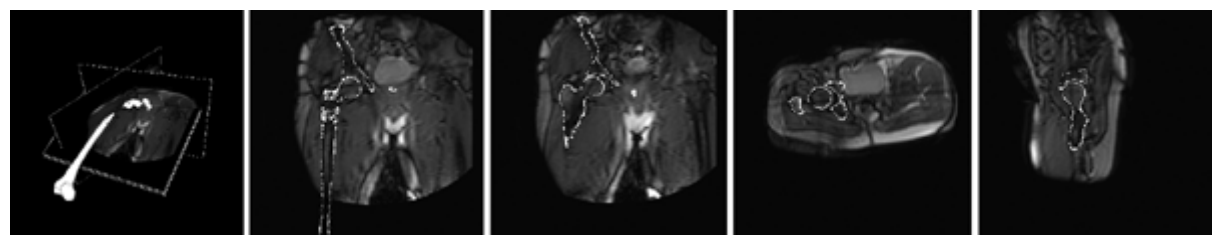

Fig. 2. 3D representation of the bones and the 4 dynamic acquisition planes, and corresponding MR images with tracked bones (in white)

\section{Conclusion and Future Work}

We present an automatic and optimized method to track bone motion from multi-slice dynamic MRI which was not previously available. This offers an accurate and non-invasive technique for the active kinematical analysis of human joints. We plan to improve the technique in terms of computational speed by using the multi-resolution approach in the optimization procedure and test the method on various movements like flexion/extension or internal/external rotation. Also, the study of the relative motion between skin markers and bones, with regards to joint angles, is under investigation. A possible application is the reduction of skin/fat sliding artifacts in optical motion capture. 
Acknowledgments. This work is supported by CO-ME (Computer Aided and Image Guided Medical Interventions, www.co-me.ch) project funded by Swiss National Research Foundation. We would like to thank Dr. H. Sadri from the orthopedic surgery department of the Southern Hospital of Fribourg for his collaboration and the Geneva University Hospital for partial support.

\section{References}

1. Lafortune, M., Cavanagh, P., Sommer, H., Kalenak, A.: Threedimensional kinematics of the human knee during walking. Journal of Biomechanics 25 (1992) 347-357

2. Magnenat-Thalmann, N., Yahia-Cherif, L., Gilles, B., Molet, T.: Hip joint reconstruction and motion visualization using mri and optical motion capture. Proceeding of the Austrian, German and Swiss society for biomedical technology congress (EMB) (2003)

3. Brossmann, J., Muhle, C., Schroder, C., Metchert, U., Bull, C., Spielmann, R., Heller, M.: Patellar tracking patterns during active and passive knee extension: evaluation with motion-triggered cine mr imaging. Radiology 187 (1993) 205-212

4. Quick, H., Ladd, M., Hoevel, M., Bosk, S., Debatin, J., Laub, G., Schroeder, T.: Real-time mri of joint movement with truefisp. Journal of Magnetic Resonance Imaging 15 (2002) 710-715

5. Brown, L.: A survey of image registration techniques. ACM Computing Surveys 24 (1992) 325-376

6. Tomazevic, D., Likar, B., Slivnik, T., Pernus, F.: 3-d/2-d registration of ct and mr to x-ray images. IEEE Transactions on Medical Imaging 22 (2003) 1407-1416

7. Zöllei, L., Grimson, E., Norbash, A., Wells, W.: 2d-3d rigid registration of xray fluoroscopy and ct images using mutual information and sparsely sampled histogram estimators. IEEE CVPR (2001)

8. Yahia-Cherif, L., Gilles, B., Moccozet, L., Magnenat-Thalmann, N.: Individualized bone modeling from mri: Application to the human hip. Proceedings of Computer Assisted Radiology and Surgery (CARS) (2003)

9. Kang, M., Sadri, H., Moccozet, L., Magnenat-Thalmann, N.: Hip joint modeling for the control of the joint center and the range of motions. Proceedings of the IFAC symposium on modelling and control in biomedical systems (2003) 23-27

10. Wu, G., Siegler, S., Allard, P., Kirtley, C., Leardini, A., Rosenbaum, D., Whittle, M., D'Lima, D., Cristofolini, L., Witte, H., Schmid, O., Stokes, I.: Isb recommendation on definitions of joint coordinate system of various joints for the reporting of human joint motion-part i: ankle,hip, and spine. Journal of biomechanics $\mathbf{3 5}$ (2002) 543-548

11. Holden, M., Hill, D., Denton, E., Jarosz, J., Cox, T., Rohlfing, T., Goodey, J., Hawkes, D.: Voxel similarity measures for 3 -d serial $\mathrm{mr}$ brain image registration. IEEE Transactions on Medical Imaging 19 (2000) 94-102

12. Shoemake, K.: Animating rotation with quaternion curves. Computer Graphics, Proceedings of SIGGRAPH 8519 (1985) 245-254

13. Roche, A., Malandain, G., Ayache, N.: Unifying maximum likelihood approaches in medical image registration. International Journal of Imaging Systems and Technology: Special Issue on 3D Imaging 11 (2000) 71-80

14. Nelder, J., Mead, R.: A simplex method for function minimization. Computer Journal 7 (1965) 308-313 